\title{
Transverse spin effects and holography
}

\author{
Oleg Teryaev ${ }^{1,2, a}$ \\ ${ }^{1}$ BLTP, JINR, 141980, Dubna, Russia \\ ${ }^{2}$ Dubna International University, 141980, Dubna,Russia
}

\begin{abstract}
The holographic sum rules for GPDs and the appearance of holographic variables in the TMD models are discussed. The relation between GPDs and TMDs in the spirit of Veneziano duality is suggested. The derivation of Burkardt sum rule for Sivers function clarifying its relation to Ji's sum rules for GPDs is presented. The possibility of its generalization for the case pf separate quark flavours implying the nodes of Sivers functions is discussed.
\end{abstract}

\section{Introduction}

The concept of holography is quite deep and multi-facet. I will address here its selected aspects related to transverse spin effects. This corresponds to the holographic sum rule for GPDs, when information about the 2 dimensional $x, \xi$ domain relevant for LO amplitudes is contained in one-dimensional section $x= \pm \xi$. In the framework of TMDs, the holography is manifested in the apperance of the varoable $x+P_{T}^{2} / M^{2} x$ combining longitudinal and transverse defrees of freedom. To relate GPDs and TMDs I discuss the sort of Veneziano-type duality between them. Also, to establsh the closer relation between Ji's sum rule for GPDs and Burkardt sum rule for Sivers function, I consider the derivation of the latter based on the properties of energy-momentum tensor.

\section{GPDs and holographic sum rules}

The analytical properties of hard exclusive amplitude result in the holographic sum rules for LO Compton formfactors

$$
\mathcal{H}(\xi)_{i}=\int_{-1}^{1} d x H_{i}(x, \xi)\left[\frac{1}{x-\xi+i \epsilon} \pm \frac{1}{x+\xi-i \epsilon}\right],
$$

where index $i$ describes the type of GPD, defining also the choice of \pm sign. For the time being we drop the dependence on the momentum transfer $t$. The representation on terms of Radon transform of Double Distributions

$H(z, \xi)=\int_{-1}^{1} d x \int_{|x|-1}^{1-|x|} d y(F(x, y)+\xi G(x, y)) \delta(z-x-\xi y)$.

allows one to get the following "holographic" sum rule

$$
\Delta \mathcal{H}=\int_{-1}^{1} d x \frac{H(x, x)-H(x, \xi)}{x-\xi+i \epsilon},
$$

\footnotetext{
ae-mail: teryaev@theor.jinr.ru
}

where the subtraction term is

$$
\Delta \mathcal{H}=\int_{-1}^{1} d x \int_{|x|-1}^{1-|x|} d y \frac{G(x, y)}{1-y} .
$$

As a result, the information on 2-dimensional $x, \xi$ region sufficient to recover LO Compton formfactor is stored in 1-dimensional section. It is this section responsible for SSA's, showing clear relation to (transverse) spin effects.

\section{Holographic variable for TMDs}

The holographic property of TMDs is manifested in the existence of the variable [2]

$$
z=x+\frac{p_{T}^{2}}{x M^{2}}
$$

implying relations between longitudinal and transverse momentum dependence. Note that its appearance in the original framework of "Zavada model" is due to the integrals (see e.g. eq. C4,C5 in [2] )

$$
\int d^{3} p \delta\left(x-\frac{p_{0}-p_{1}}{M}\right) \ldots
$$

which may be considered as a 3-dimensional Abel transform, and as usual at the odd numers of dimensions the inverse transform has a simple local form. From the other side, this variable is rooted in the Lorentz [3] and,in particular, rotational [4] symmetries. One may wonder whether these symmetries are related to the appearance of 2-dimensional Abel transform for twist-3 GPDs[5].

\section{GPDs and TMDs: $s \leftrightarrow t$ duality?}

It would be interesting to look for common origins of GPDs and TMDs holographic properties. Probably they may correspond to the respective properties of Wigner 
function [6] being their "common ancestor". At the same time, the "horizontal" relations are certainly of interest. One may for this purpose consider TMDs as a hadron parton amplitudes with (unphysical)

$$
s \sim-p_{T}^{2}
$$

which may be corrected by mass term so that

$$
s \approx x^{2} M^{2}-p_{T}^{2}
$$

GPDs dependence on $t$ and TMDs dependence on $p_{T}^{2}$ may be considered as a $s$ and $t$ dependence of the same hadron-parton amplitude. It would be interesting to look for dual Veneziano-type parameterization for these dependencies. It may be supported by the light-cone models [6, 7], when GTMDs correspond to the overlap of wave functions of the type

$$
\bar{\Psi}\left(p_{T}+q_{T} / 2\right) \Psi\left(p_{T}-q_{T} / 2\right)
$$

where $q_{T}^{2}=-t$ and reflection symmetry implies the symmetry w.r.t. interchange $q_{T} \leftrightarrow p_{T}$.

\section{Burkardt sum rule(s) and energy-momentum tensor}

The relations between GPDs and TMDs may be also reflected in the relations between respective sum rules. The interesting example is provided by Ji's sum rules for GPDs and Burkardt sum rule (BSR) for Sivers TMD. The similarity of these sum rules was conjectured earlier [8] in connection with equivalence principle for spin-gravity interaction.

The most straightforward way to test this conjecture would be to derive the BSR relying on the properties of energy-momentum tensor, like the Ji's SR and equivalence principle. For this reason one may use the relation of the relevant moment of Sivers function to the twist-3 matrix element [10]. Expressing the matrix element of quark energy-momentum tensor in terms of gluonic pole [11] strength $T\left(x_{1}, x_{2}\right)$ and taking into account that the forward matrix element is fixed by energy-momentum conservation one get

$$
\sum \int_{-1}^{1} d x_{1} d x_{2} \frac{T\left(x_{1}, x_{2}\right)}{x_{1}-x_{2}}=0 .
$$

Note that the sum should include gluons as well as quarks, and the relation of the moment of Sivers function to the gluonic twist-3 matrix element is not yet known. We anticipate its existence and in the meantime will discuss the quark contributions.

The pole in the denominator appears due to the expression of matrix element with gluon covariant derivative in terms of that of gluonic field strength and, naively, should be treated in principal value sense. In that case, $T\left(x_{1}, x_{2}\right)$ is the symmetric function of its variables due to T-invariance [10] and 1.h.s of (5) is identically zero, so that no sum rule emerges.
However, the arguments based on electromagnetic gauge invariance imply [12] that the similar pole appearing in the twist 3 SSA in Drell-Yan process acquires the imaginary part. Incorporating it into the sum rule

$$
\sum \int_{-1}^{1} d x_{1} d x_{2} \frac{T\left(x_{1}, x_{2}\right)}{x_{1}-x_{2}+i \epsilon}=0,
$$

one get the symmetric imaginary part of the integrand effectively violating T-invariance resulting in the sum rule:

$$
\sum \int_{-1}^{1} d x T(x, x)=0 .
$$

One can see that Burkardt sum rule requires, besides the energy-momentum conservation, some dynamical information, like in its original derivation.

One may wonder, whether this sum rule may be valid for each quark flavour (and gluon) separately. The structure in front of twist 3 vector correlator

$$
\epsilon^{\mu S_{T} P n}
$$

contains the gauge and transverse direction fixing vector $n$. If gauge invariant decomposition of energy-momentum tensor is possible, the sum rule for each quark (combined with antiquark) flavour $i$ should be valid

$$
\int_{-1}^{1} d x T_{i}(x, x)=0 .
$$

This implies the nodes [13] of Sivers function anticipated at the first "Transversity 2005" conference at Como [14].

\section{Acknowledgments}

I am indebted to Umberto d'Alesio and Francesco Murgia for warm hospitality at Sardinia.

This work was supported in part by RFBR grants 1302-01060, 14-01-00647,12-02-00613,12-02-91526.

\section{References}

[1] O. V. Teryaev, hep-ph/0510031.

[2] A. V. Efremov, P. Schweitzer, O. V. Teryaev and P. Zavada, Phys. Rev. D 80 (2009) 014021 [arXiv:0903.3490 [hep-ph]]. ibid., 83 (2011) 054025 [arXiv:1012.5296 [hep-ph]].

[3] U. D'Alesio, E. Leader and F. Murgia, Phys. Rev. D 81 (2010) 036010 [arXiv:0909.5650 [hep-ph]].

[4] C. Lorce and B. Pasquini, Phys. Rev. D 84 (2011) 034039 [arXiv:1104.5651 [hep-ph]].

[5] A. M. Moiseeva and M. V. Polyakov, Nucl. Phys. B 832 (2010) 241 [arXiv:0803.1777 [hep-ph]].

[6] B. Pasquini and C. Lorce, Few Body Syst. 55 (2014) 287.

[7] D. Muller and D. S. Hwang, arXiv:1407.1655 [hepph].

[8] O. V. Teryaev, AIP Conf. Proc. 915 (2007) 260 [hep$\mathrm{ph} / 0612205]$. 
[9] D. Boer, P. J. Mulders and F. Pijlman, Nucl. Phys. B 667 (2003) 201 [hep-ph/0303034].

[10] A. V. Efremov and O. V. Teryaev, Sov. J. Nucl. Phys. 39 (1984) 962 [Yad. Fiz. 39 (1984) 1517]; Phys. Lett. B 150 (1985) 383.

[11] J. w. Qiu and G. F. Sterman, Phys. Rev. Lett. 67 (1991) 2264.
[12] I. V. Anikin and O. V. Teryaev, Phys. Lett. B 690 (2010) 519 [arXiv:1003.1482 [hep-ph]].

[13] D. Boer, Phys. Lett. B 702 (2011) 242 [arXiv:1105.2543 [hep-ph]].

[14] O.V, Teryaev, in Transversity 2005, ed. by V. Barone and P.G. Ratcliffe (World Scientific, 2006) p.276-283. 